\title{
Efficacy and safety outcomes in randomized controlled trials investigating hydroxychloroquine for COVID-19
}

\author{
Daniela Junqueira ${ }^{1}$ and Brian Rowe ${ }^{1}$ \\ ${ }^{1}$ University of Alberta
}

May 21, 2020

\begin{abstract}
Aims: To assess whether randomized clinical trials (RCTs) proposed to evaluate treatment of COVID-19 with HQ or chloroquine include outcome definitions and data collection plans to produce meaningful efficacy/effectiveness and safety outcomes. Methods: We searched the World Health Organization International Clinical Trials Registry Platform (WHO-ICTRP) database for registers of RCTs evaluating HQ or chloroquine, alone or in any combination, to treat patients diagnosed with COVID-19 compared with any other treatment option. The final search was performed on April 8th, 2020. Results: Among 51 registered RCTs (median sample size of 262; IQR: 100, 520), 34 (67\%) reported a clinical outcome, 12 (24\%) a surrogate outcome, and five $(10 \%)$ a combination of clinical and surrogate outcomes as primary endpoints. Clinical status/recovery and all-cause mortality/mortality accounted for $49 \%$ of the unique domains among 20 different clinical outcome domains of efficacy. Twenty-four (47\%) RCTs did not describe plans to assess safety outcomes; when assessed, safety outcomes were determined in generic terms of total, severe or serious adverse events. Conclusions: The RCTs investigating HQ or chloroquine include heterogenous and insufficient approaches to measure efficacy/effectiveness and safety that are relevant to patients and clinical practice. These findings provide important insights to inform clinical and regulatory decisions that can be drawn about the efficacy/effectiveness and safety of these agents in patients with COVID-19.
\end{abstract}

\section{What is already known about this subject}

- There is an urgent need for a treatment that reduce the morbidity and mortality of patients diagnosed with COVID-19;

- Chloroquine, and its derivate hydroxychloroquine (HQ), have been popular potential therapies described in the scientific literature and social media.

\section{What this study adds}

- The results show that randomized controlled trials (RCTs) that are being planned to investigate the efficacy/effectiveness of HQ or chloroquine include a heterogeneous set of clinical outcomes domains;

- The planned RCTs include insufficient and unstructured approaches to detect adverse events that are relevant to patients and to inform clinical practice;

- This study highlights three critical areas that can be improved to better inform clinical and regulatory decisions about the value of $\mathrm{HQ}$ or chloroquine in the treatment of COVID-19: selection of efficacy/effectiveness outcomes, assessment of treatment harms and patient safety, reducing missing and variable dosing schedules and treatment duration.

\section{Introduction}

On December 31, 2019, a cluster of cases of pneumonia of unknown etiology was reported in the city of Wuhan, China, and later identified as being caused by a novel coronavirus. 1 The ability of the novel Severe Acute Respiratory Syndrome CoronaVirus-2 (SARS-CoV-2; hereafter referred to 
as COVID-19) ${ }^{2}$ to infect human hosts and be transmitted among individuals rapidly evolved into a global outbreak with 2.2 million confirmed cases in 211 countries as of April 17, 2020, 18:00 GMT-6 (https://www.who.int/emergencies/diseases/novel-coronavirus-2019). Though the majority ( $20 \%$ ) of the confirmed COVID-19 cases develop a mild condition, a smaller percentage ( $15 \%)$ of patients with confirmed COVID-19 require hospitalization and some develop a severe condition $(\sim 5 \%)$ that require mechanical ventilation in the first 24 hours of hospital admission. ${ }^{3}$ Clinical complications such as profound acute hypoxemic respiratory failure and sepsis requiring vasopressor treatment ${ }^{4,5}$ have led to a total number of 153,177 deaths worldwide (https://www.who.int/emergencies/diseases/novel-coronavirus-2019; last updated April 17, 2020, 18:00 GMT-6).

Worldwide, the capacity of the healthcare systems to offer care for patients diagnosed with COVID-19 depends on ICU beds and emergency departments (ED) capability to accommodate the additional care requirements brought by the pandemic. This is of critical relevance considering the high ICU occupancy commonly seen in many locations ${ }^{6}$ and the long-recognized ED overcrowding and its negative consequences on patient outcomes ${ }^{7-9}$. Moreover, the current management of patients with COVID-19 is supportive, and recovery time is estimated at around three to six weeks for critically ill patients. ${ }^{10} \mathrm{In}$ this challenging scenario, it is not surprising that, following the recognition of COVID-19 as a Public Health Emergency of International Concern on January, 30, 2020, (https://www.who.int/emergencies/diseases/novel-coronavirus-2019/eventsas-they-happen), there has been a frantic search for effective treatments. On April 6, 2020, there were 788 entries of COVID-19 trials registered on the World Health Organization International Clinical Trials Registry Platform (WHO-ICTRP), with more expected.

Clinical trials provide vital evidence to establish the efficacy and safety of new medicines or new indications for existing medicines. To be informative, however, they have to be designed and implemented with appropriate standards to provide meaningful evidence. ${ }^{11}$ Meaningful evidence fundamentally includes the definition of outcomes that reflect efficacy/effectiveness (beneficial treatment effect) and safety (effects of the treatment that may be harmful to patients). ${ }^{12}$ Efficacy/effectiveness outcomes should represent clinically meaningful results that directly measure how a patient feels, functions, or survives. ${ }^{13}$ Alternatively, trials may use surrogate outcomes instead of clinical ones. Trials assessing surrogate outcomes may be completed faster and be less expensive. However, surrogate outcomes may or may not predict clinical results and translate in meaningful evidence of efficacy/effectiveness. ${ }^{13,} 14$

Likewise, safety outcomes are essential in defining the value of a treatment intervention for healthcare providers, patients, and health systems. Despite the importance of finding a treatment that is effective in mitigating or curing patients diagnosed with COVID-19, it is critical to appropriately define and detect the potential adverse events of the treatment options under investigation. There are guidance and legal requirements for protocols of clinical trials to plan the data collection of adverse events, whether applying systematic or non-systematics assessment approaches ${ }^{12,15,16}$. The objective of this study was to assess whether the randomized clinical trials (RCTs) registered on the WHO-ICTRP for patients diagnosed with COVID-19 include definitions and data collection plans to produce evidence on meaningful efficacy, effectiveness and safety outcomes.

\section{Methods}

We selected as eligible studies parallel RCTs evaluating either hydroxychloroquine (HQ) or chloroquine to treat patients diagnosed with COVID-19, used alone or in any combination, and compared with any other treatment option (including placebo). We selected HQ and chloroquine because these drugs have received widespread support as effective treatments for patients diagnosed with COVID-19 infection. These claims arose following demonstration of in vitro viral activity against SARS-CoV-2, and with potential viral load reduction in a case series report. ${ }^{17,}{ }^{18}$ In the United States, the FDA issued an emergency use authorization to allow the use of these drugs in adolescents and adults hospitalized for COVID-19 not participating in clinical trials. ${ }^{19}$ Moreover, social media and leadership support, lead to widespread shortages of the medication. Traditionally, these immune-suppressants have been used to treat autoimmune diseases such as rheumatoid arthritis and inflammatory bowel disease; however, they have also been approved for the treatment of malaria 
since $1955 .{ }^{20}$ Familiarity and potential benefit have created a push for expedited clinical trials. We included trials recorded in the clinical trial register of any country and at any recruitment status. Uncontrolled trials and observational studies were excluded.

We downloaded the COVID-19 WHO-ICTRP database (https://www.who.int/ictrp/search/en/) on April $8^{\text {th }}, 2020$ at 10:30 GMT-6. On the database, we filtered studies according to the intervention (HQ or chloroquine) and the study design (randomized versus non-randomized). All the retrieved registers were considered included and reviewed for data extraction purposes. Patients and investigators were not aware of this study at the time of their submissions.

Data extraction and sources

One author (DJ) extracted data using a pre-standardized form. Quality control was performed by reextracting data from $15 \%$ of the included trial registers. Information on the trial ID, scientific title, date of registration, recruitment status, patient population and funding sources were extracted from the WHOICTRP database. Information on the country where the trials were planned to be conducted was extracted primarily from the WHO-ICTRP database and completed using the trial register data when appropriate.

The trial's register was accessed and provided addition information to characterize the RCTs according to:

- Number of participants planned to be recruited;

- Age and sex of the participants planned to be recruited;

- Intervention and comparison treatments, including doses and administration schedules;

- Treatment duration;

- Efficacy/effectiveness outcomes defined as primary endpoints;

- Safety outcomes, i.e., adverse events;

- Timeframe for the assessment of the efficacy/effectiveness and safety outcomes;

- Mode data collection of the safety outcomes.

The efficacy/effectiveness outcomes were classified as clinical (e.g., improvement or recovery of respiratory symptoms) or surrogate outcomes (e.g., viral load, biomarkers, etc.). The mode of data collection of the adverse events was classified as "systematic assessment" when specific ascertaining methods to detect the occurrence of adverse events were described by the use of checklists, questionnaires, or laboratory tests at regular intervals, and as "non-systematic assessment" when the detection methods relied on the spontaneous report of adverse events by clinicians or participants ${ }^{15}$.

Data analysis

Characteristics of the design of the RCTs were summarized according to the number trial registers documenting clinical or surrogate outcomes and describing data ascertainment methods to detect adverse events. Simple proportions were reported for dichotomous outcomes. For continuous data, means and standard deviations (SD) or medians and interquartile ranges (IQR) were reported, as appropriate. Comparisons between continuous variables were made using t-test and reporting the mean difference (MD) with $95 \%$ confidence intervals (CI).

\section{Results}

Among 927 clinical trial registers recorded on the WHO-ICTRP database, 72 registers were identified as RCTs investigating the use of HQ or chloroquine for COVID-19 infection and considered potentially eligible to this study (Figure 1). Among these 72 registered trials, two were identified as duplicated entries of the same trial registered in more than one clinical trial register, seven trials have been cancelled, and 12 were trials testing prophylaxis treatments. Therefore, 51 clinical trial registers were included for analysis.

Table 1 summarizes the characteristics of the clinical trials planned to investigate the use of HQ or chloroquine to treat patients diagnosed with COVID-19. The proposal of RCTs to test the hypothesis of whether these drugs could be beneficial for people with COVID-19 started in February 2020 when 12 trials were registered. In the following month of March, the number of trials registered tripled. All trials planned to include adults 
of both sexes, and three trials (6\%) also planned to include adolescents. A total of 27 registered RCTs (53\%) were not yet actively recruiting patients.

The proposed dosing schedule and treatment duration varied among the trials. Seventeen trials reported at least one arm with a fixed dosing administration schedule of $\mathrm{HQ}$ or chloroquine ranging from a daily amount of 200 to $1,200 \mathrm{mg}$ (Table 2). One trial planned to administer one single dose of HQ (200 mg) in combination with other drugs. Considering the dosing schedule of all treatment arms of either HQ or chloroquine, maximum treatment duration described ranged from seven days to 14 days. Twenty trials reported at least one arm with a variable dosing administration schedule of HQ or chloroquine (Table 3). Considering all treatment arms with a variable dosing schedule, treatment duration varied from 5 to 16 days. Fourteen registered trials did not report information on the treatment duration. One trial (2\%) reported plans to monitor adherence and two trials (4\%) reported funding support from sources with potential commercial interest (data not shown).

$<<$ Table $1>>$

$<<$ Table $2>>$

$<<$ Table $3>>$

Forty-five of the RCTs (90\%) were planned to be conducted in one single country (Supplement, Table S1) with a mean sample size of 262 (IQR: 100, 520). Among the trials planned to be implemented in one single country, China was the main location $(16 ; 32 \%)$ followed by the United States $(5 ; 10 \%)$. Five (10\%) of the registered RCTs were designed to be conducted in multiple countries; one trial register did not provide information on the location where the RCT was planned to be implemented. Overall, the proposed clinical trials anticipate recruiting a total of 37,303 participants, among outpatients and inpatients, to be randomized to receive a variety of experimental and comparison treatments with HQ, chloroquine or other agents in diverse combinations and dose schedules (Supplement, Table S2). Only fourteen (27\%) of the trial registers reported the number of patients being recruited to the treatment and comparison arms; among these trials, a total of 1,138 patients would receive $\mathrm{HQ}$ or chloroquine alone or in combination with other drugs (data not shown).

Table 4 summarizes the type of outcomes described in the registry of the RCTs and the related assessment timeframe. One-third of the clinical trials included in their registered information a surrogate outcome to be measured as a primary endpoint; the remaining trials $(34 ; 67 \%)$ described plans to assess one clinical outcome as a primary endpoint. The timeframe of outcome assessment varied substantially among the designs of the RCTs. Trials planning to measure only clinical efficacy/effectiveness outcomes described timeframes of assessment ranging from 5 to 120 days (median 15; IQR: 15, 28). Trials planning to measure only surrogate outcomes defined timeframes of assessment ranging from 3 to 56 days (median 15; IQR: 15, 28). The RCTs planning to evaluate a clinical outcome described longer timeframes for outcome assessment in comparison with trials planning to assess a surrogate outcome (MD 6.3; 95\% CI: -10.51 to $23.12 ; \mathrm{P}=0.45$ ). Among all 51 registered RCTs describing at least one clinical or surrogate efficacy/effectiveness outcome, 13 (26\%) did not report a timeframe for outcome assessment.

Twenty-four $(47 \%)$ of the registered RCTs did not describe plans to assess a single safety outcome. Among the trials including a description of at least one safety outcome $(n=28)$, most $(25 ; 89 \%)$ did not report the method to be implemented for the detection of adverse events. The timeframe for the assessment of safety outcomes was not defined in $13(46 \%)$ of the trials reporting plans to measure at least one safety outcome. The timeframe for the assessment of the safety outcomes ranged from 7 to 120 days (median 28; IQR: 14, 30). The timeframe of safety outcome assessment was planned to be longer in comparison with the assessment of clinical outcomes (MD -9.8; $95 \%$ CI: -26.08 to $6.56 ; \mathrm{P}=0.23$ ).

$<<$ Table $4>>$

Figure 2 describes the domains of the outcomes defined in the RCTs assessing the efficacy/effectiveness of HQ or chloroquine in the treatment of patients diagnosed with COVID-19. Twenty different clinical 
outcomes were described among trials with at least one clinical efficacy/effectiveness outcome defined in the trial registration. Clinical status/recovery and all-cause mortality/mortality accounted for $49 \%$ of the unique clinical outcome domains proposed to assess the efficacy/effectiveness of HQ or chloroquine treatment on patients diagnosed with COVID-19. Twenty-one different surrogate outcomes were identified in the registered RCTs planning to measure at least one surrogate outcome, with viral load and virologic clearance accounting for $36 \%$ of the surrogate outcomes identified.

Figure 3 summarizes the domains of the safety outcomes described to detect the potential harms of the treatment with hydroxychloroquine or chloroquine. The generic terminologies total, severe and serious adverse events accounted for $68 \%$ of the unique domains reported in at least two trial registers. Twentythree different domains were reported by only one the registers of the clinical trials where the assessment of at least one safety outcome was identified.

$<<$ Figure $2>>$

$<<$ Figure $3>>$

\section{Discussion}

Clinical trials are study designs central to the regulatory and commercialization process of therapeutic interventions such as pharmaceutical agents and devices. Regulatory decisions informed by clinical trials data often represents a certificate of clinical and safety value to new medicines or new indications of existing medicines. ${ }^{21}$ Given the potential severity of the COVID-19 infection, the need to find a mitigating or curative treatment is beyond urgent. Several candidate compounds addressing different disease processes (e.g., antibiotics, anti-viral, immune-suppressants, anticoagulants, oxygen delivery, etc.) have been proposed and are now undergoing clinical trials. Both $\mathrm{HQ}$ and chloroquine have been popular potential therapies described in the scientific literature and social media, and evidence for their efficacy/effectiveness and safety are desperately needed. Nevertheless, one recent evaluation of the three published HQ trials found important methodological weaknesses and sub-optimal reporting of key information. ${ }^{22}$

In this study, we found that RCTs proposed to evaluate the clinical efficacy/effectiveness and safety of HQ or chloroquine in the treatment of patients diagnosed with COVID-19 are designed to collect data that vary substantially in terms of the outcome domain used to determine the evidence base upon which these drugs will be judged. Moreover, data on safety outcomes are overlooked or only superficially included among the outcomes planned to be measured in these trials. Finally, essential information related to dosing schedules, treatment duration and timeframe of outcome assessment were frequently missing in the description of the RCTs. Overall, this analysis yielded three major areas of concern.

\section{Selection of efficacy /effectiveness outcomes}

The outcomes measured in clinical trials are critical in providing meaningful data and in allowing comparison among the results of other RCTs and different interventions. ${ }^{23}$ Though most of the evaluated RCTs specified at least one clinical outcome as a primary endpoint, the outcome domains varied widely. For example, while half of the registered trials described plans to assess all-cause mortality/mortality and clinical status/recovery to evaluate the efficacy/effectiveness of the drugs, the remaining trials defined over 15 different outcome domains. Ideally, the results of RCTs are subsequently combined in systematic reviews and meta-analysis, which are vital to informing all healthcare providers and health decision-makers. This may be particularly true during the COVID-19 pandemic, as many of the existing trials are small and a definitive, mega-trial trial may not emerge from the list identified. In the presence of highly heterogeneous outcomes, the development of systematic reviews and meta-analysis is likely less informative if not precluded.

During the writing of this study, a set of core outcomes of relevance to be measured in studies including adult hospitalized patients diagnosed with COVID-19 was released (http://www.cometinitiative.org/Studies/Details/1538). The core outcome set comprised mortality and respiratory support, outcome domains included in some, but not all, RCTs planned to evaluate the effect of HQ or chloroquine 
in patients diagnosed with COVID-19. More concerning, several of the clinical trials registered in the WHOICTRP database included only surrogate outcomes to estimate the efficacy/effectiveness of the drugs. There exist several examples where positive results in trials measuring surrogate outcomes were not replicated in efficacy/effectiveness trials where clinical outcomes were measured. ${ }^{14,} 24$

To increase research usefulness and relevance to patients and the health system, any investigation on drugs that might potentially treat patients diagnosed with COVID-19 needs to include a minimal standardized set of clinical outcomes of efficacy/effectiveness. This set of outcomes will likely be informed by the evolving research on the clinical characteristics of this new disease. Ultimately, the strain on capacity on the health system will only abate when we identify treatments that improve patients' clinical outcomes, such as the reduction of intubation rates and subsequent deterioration. The development of a safe, effective, and widely available vaccine will be the long-term solution to the COVID-19 pandemic.

\section{Inadequate assessment of treatment harms and patient safety}

Chloroquine, and its derivate HQ, are antimalarial drugs; HQ is approved by the Food and Drug Administration (FDA) since $1955^{20}$ and it is also important in the treatment of immune-mediated diseases such as lupus erythematosus and rheumatoid arthritis. ${ }^{25}$ Both drugs are known to induce irreversible retinal damage, cardiomyopathy and QTc prolongation, severe hypoglycemia and dermatologic adverse events. ${ }^{20}$ The severity of these adverse effects range from mild to severe; occasionally these agents have been found to cause death.

Since we can anticipate a set of adverse events that are highly relevant to patients and clinical practice, the proposal of RCTs should contain plans to systematically assess fully defined adverse events according to appropriate timeframes. ${ }^{12,16,26}$ For instance, QTc prolongation and drug-induced arrhythmias like torsades de pointes, are of concern in critically ill patients with COVID- $19^{27}$ and should, therefore, be carefully ascertained. Monitoring QTc through electrocardiographic tracings on a regular basis would represent a systematic approach to the problem; even if monitoring is required to be performed remotely for safety reasons. The systematic assessment of adverse events can improve the accuracy of estimates within trials ${ }^{28}$ while also minimizing bias. ${ }^{29}$ Finally, the assessment of defined anticipated adverse events, together with their seriousness, severity and duration, would be more informative than the mere documentation of generic events.

In this study, we showed that retinopathy, cardiac and dermatologic adverse events, and hypoglycemia were planned to be assessed in a single clinical trial among the 51 trials that had been registered to evaluate the treatment with HQ or chloroquine for patients diagnosed with COVID-19. Outcomes of safety were not included among the outcomes defined in several of the proposed trials $(24,47 \%)$, while the remaining RCTs reported a non-specific approach of observation of safety outcomes. Based on these results, and the fact the many adverse effects are rare in small clinical trials, we are concerned that the evidence on the harms of these investigational drugs to patients diagnosed with COVID-19 may likely be incomplete and biased.

\section{Missing information}

The comprehensive and prospective registration of clinical trials has been internationally supported since 2004 as a way to reduce the selective publication of studies and the selective reporting of outcomes. ${ }^{30}$ Since the early years of clinical trial registration, ensuring that the registered data are complete and accurate has been a challenging objective of multiple enforcement mechanisms, including legal requirements. ${ }^{26,}{ }^{30}$ Remarkably, approximately one-third of the registered RCTs included in this study had at least one piece of missing information, either related to treatment dose, duration, timeframes of outcome assessment or the lack of definition of a safety outcome. This is of particular concern amid the current pandemic scenario where the rush to test any potential helpful drug may pose a risk that low-quality evidence may be used to support clinical decisions with unpredictable impacts to patients and the health system.

\section{Limitations}

We reviewed the information provided by all clinical trials focused on $\mathrm{HQ}$ and chloroquine for patients 
diagnosed with COVID-19 available in the WHO-ICTRP database until April $8^{\text {th }}$, 2020, at 10:30 GMT6. We chose the WHO-ICTRP because it compiles data from ClinicalTrials.gov and 10 primary registers (https://www.who.int/ictrp/en/). In the scenario of the COVID-19 pandemic, we judged that this approach would provide an improved overview of clinical trials being planned in different countries. Nevertheless, as we did not search the primary registers directly, it is possible that some potentially eligible registered RCTs could have been missed. Also, the data analyzed in this study is limited to the information provided in the record of the clinical trials' register. A minority of the clinical trials provided access to the full study protocol; therefore, the data collected and analyzed in this study may be incomplete if authors have deliberately left information missing on the publicly available record of the planned trials. Nevertheless, if the trial registers are incomplete for low quality designs or deliberate actions, this represents a risk to the strength of the evidence that will ultimately be available to inform decisions. Finally, as the development of research on COVID-19 is evolving fast, additional RCTs may exist which were planned but registered after the study timeline; these trials were not included in the present analysis.

\section{Conclusion}

There is an increasing number of RCTs being planned to evaluate the clinical efficacy/effectiveness and safety of HQ or chloroquine in the treatment of patients diagnosed with COVID-19. Outcomes domains described in these clinical trials are highly heterogeneous and include clinical and/or surrogate measures. Moreover, despite $\mathrm{HQ}$ and chloroquine being known to induce cardiovascular and other adverse events that can be irreversible and potentially life-threatening, the registered RCTs do not describe systematic assessment methods to accurately detect adverse events. The pandemic scenario is demanding researchers to register, plan and deploy RCTs at an incredibly fast pace. The present analysis supports the need for improvements in the design of ongoing and future RCTs. Ultimately, finding safe and effective treatments is required to decrease the burden on patients, providers and health care systems worldwide created by patients diagnosed with COVID-19.

\section{Authorship}

Dr. Junqueira led the research conceptualization, data curation, formal analysis, investigation, methodology, project administration and visualization. She wrote the original draft and worked with Dr. Rowe on reviewing and editing the final manuscript.

Dr. Rowe led the funding acquisition and worked with Dr. Junqueira on the research conceptualization, analysis, and reviewing and editing the final manuscript.

\section{Funding Sources/Disclosures}

Dr. Rowe's research is supported by the Canadian Institutes of Health Research (CIHR) through the Government of Canada (Ottawa, ON) by a CIHR Scientific Directors Operating Grant (SOP 168483). The funders had not role in the study design, data collection, analysis, and interpretation of data; in the writing of the report; and in the decision to submit the article for publication.

Dr. Junqueira receives salary and employee benefits from the Emergency Medicine Research Group (EMeRG) at the University of Alberta. She has no competing interests to declare.

\section{Transparency statement}

Dr. Junqueira and Dr. Rowe (the manuscript's guarantors) affirm that the manuscript is an honest, accurate, and transparent account of the study being reported.

\section{Ethical approval}

Not applicable.

\section{Data sharing statement}


The data used for analysis is in the public domain. Nevertheless, the full dataset analyzed can be requested from the authors.

\section{References}

1. World Health Organization (WHO). Novel Coronavirus (2019-nCoV), Situation Report - 1, 21 January 2020 . WHO; 2020. https://www.who.int/docs/default-source/coronaviruse/situation-reports/20200121sitrep-1-2019-ncov.pdf?sfvrsn=20a99c10_4

2. Gorbalenya AE, Baker SC, Baric RS, et al. Severe acute respiratory syndrome-related coronavirus $</$ em $>$ : The species and its viruses - a statement of the Coronavirus Study Group.bioRxiv . 2020:2020.02.07.937862. doi:10.1101/2020.02.07.937862

3. Mahase E. Covid-19: most patients require mechanical ventilation in first 24 hours of critical care. $B M J$ . Mar 24 2020;368:m1201. doi:10.1136/bmj.m1201

4. Huang C, Wang Y, Li X, et al. Clinical features of patients infected with 2019 novel coronavirus in Wuhan, China. Lancet . Feb 15 2020;395(10223):497-506. doi:10.1016/S0140-6736(20)30183-5

5. Bhatraju PK, Ghassemieh BJ, Nichols M, et al. Covid-19 in Critically Ill Patients in the Seattle Region - Case Series. N Engl J Med. Mar 30 2020;doi:10.1056/NEJMoa2004500

6. Grasselli G, Pesenti A, Cecconi M. Critical Care Utilization for the COVID-19 Outbreak in Lombardy, Italy: Early Experience and Forecast During an Emergency Response. JAMA . Mar 13 2020;doi:10.1001/jama.2020.4031

7. Kirkland SW, Soleimani A, Rowe BH, Newton AS. A systematic review examining the impact of redirecting low-acuity patients seeking emergency department care: is the juice worth the squeeze? Emerg Med $J$. Feb 2019;36(2):97-106. doi:10.1136/emermed-2017-207045

8. Rowe BH, Guo X, Villa-Roel C, et al. The role of triage liaison physicians on mitigating overcrowding in emergency departments: a systematic review. Acad Emerg Med . Feb 2011;18(2):111-20. doi:10.1111/j.15532712.2010.00984.x

9. Morley C, Unwin M, Peterson GM, Stankovich J, Kinsman L. Emergency department crowding: A systematic review of causes, consequences and solutions. PLoS One . 2018;13(8):e0203316. doi:10.1371/journal.pone.0203316

10. World Health Organization Director. General's opening remarks at the media briefing on COVID-19 24 February 2020 . 2020. Accessed 07 April 2020. https://www.who.int/dg/speeches/detail/who-directorgeneral-s-opening-remarks-at-the-media-briefing-on-covid-19 - 24-february-2020

11. Zarin DA, Goodman SN, Kimmelman J. Harms From Uninformative Clinical Trials. JAMA. Jul 25 2019; doi:10.1001/jama.2019.9892

12. Chan AW, Tetzlaff JM, Gotzsche PC, et al. SPIRIT 2013 explanation and elaboration: guidance for protocols of clinical trials. BMJ . Jan 8 2013;346:e7586. doi:10.1136/bmj.e7586

13. Food and Drug Administration. Surrogate Endpoint Resources for Drug and Biologic Development . 2018. Accessed 10 April 2020. https://www.fda.gov/drugs/development-resources/surrogate-endpointresources-drug-and-biologic-development

14. Fleming TR, DeMets DL. Surrogate end points in clinical trials: are we being misled? Ann Intern Med . Oct 1 1996;125(7):605-13. doi:10.7326/0003-4819-125-7-199610010-00011

15. Food and Drug Administration. Amendments Act of 200\%. Public Law 110-85.

16. Zarin DA, Tse T, Williams RJ, Califf RM, Ide NC. The ClinicalTrials.gov results database-update and key issues. N Engl $J$ Med . Mar 3 2011;364(9):852-60. doi:10.1056/NEJMsa1012065 


\subsection{6/NEJMsa1012065.}

17. Wang M, Cao R, Zhang L, et al. Remdesivir and chloroquine effectively inhibit the recently emerged novel coronavirus (2019-nCoV) in vitro. Cell Res . Mar 2020;30(3):269-271. doi:10.1038/s41422-020-0282-0

18. Gautret P, Lagier JC, Parola P, et al. Hydroxychloroquine and azithromycin as a treatment of COVID19: results of an open-label non-randomized clinical trial. Int J Antimicrob Agents . Mar 20 2020:105949. doi:10.1016/j.ijantimicag.2020.105949

19. Food and Drug Administration. Request for Emergency Use Authorization For Use of Chloroquine Phosphate or Hydroxychloroquine Sulfate Supplied From the Strategic National Stockpile for Treatment of 2019 Coronavirus Disease . 2020. Accessed 07 April 2020. https://www.fda.gov/media/136534/download

20. Food and Drug Administration. Drugs@FDA: FDA-Approved Drugs: Plaquenil . Accessed 07 April 2020. https://www.accessdata.fda.gov/scripts/cder/daf/index.cfm?event=overview.process\&ApplNo=009768

21. Lehoux P, Miller FA, Daudelin G, Denis JL. Providing Value to New Health Technology: The Early Contribution of Entrepreneurs, Investors, and Regulatory Agencies. Int J Health Policy Manag . Sep 1 2017;6(9):509-518. doi:10.15171/ijhpm.2017.11

22. Alexander PE, Debono VB, Mammen MJ, et al. COVID-19 research has overall low methodological quality thus far: case in point for chloroquine/hydroxychloroquine. Journal of Clinical Epidemiology . 2020;doi:10.1016/j.jclinepi.2020.04.016

23. Williamson PR, Altman DG, Bagley H, et al. The COMET Handbook: version 1.0. Trials . Jun 20 2017;18(Suppl 3):280. doi:10.1186/s13063-017-1978-4

24. Bikdeli B, Punnanithinont N, Akram Y, et al. Two Decades of Cardiovascular Trials With Primary Surrogate Endpoints: 1990-2011.J Am Heart Assoc . Mar 21 2017;6(3)doi:10.1161/JAHA.116.005285

25. Yazdany J, Kim AHJ. Use of Hydroxychloroquine and Chloroquine During the COVID-19 Pandemic: What Every Clinician Should Know. Ann Intern Med . Mar 31 2020;doi:10.7326/M20-1334

26. Zarin DA, Tse T, Williams RJ, Carr S. Trial Reporting in ClinicalTrials.gov - The Final Rule. $N$ Engl $J$ Med . Nov 17 2016;375(20):1998-2004. doi:10.1056/NEJMsr1611785

27. CPS [Internet]. Ottawa (ON): Canadian Pharmacists Association; c2016 [updated 2020 April; cited 2020 April 15]. Gleevec [Hydroxychloroquine]. Available from: http://www.e-cps.ca or http://www.myrxtx.ca. Also available in paper copy from the publisher.

28. Mayo-Wilson E, Fusco N, Li T, et al. Harms are assessed inconsistently and reported inadequately part 1: systematic adverse events. J Clin Epidemiol . Sep 2019;113:20-27. doi:10.1016/j.jclinepi.2019.04.022

29. Mayo-Wilson E, Fusco N, Hong H, Li T, Canner JK, Dickersin K. Opportunities for selective reporting of harms in randomized clinical trials: Selection criteria for non-systematic adverse events. Trials . Sep 5 2019;20(1):553. doi:10.1186/s13063-019-3581-3

30. Ghersi D, Pang T. From Mexico to Mali: four years in the history of clinical trial registration. J Evid Based Med . Feb 2009;2(1):1-7. doi:10.1111/j.1756-5391.2009.01014.x

\section{Tables and Figures}

Table 1 Characteristics of the randomized controlled trials for the treatment of COVID-19 with hydroxychloroquine or chloroquine $(\mathrm{n}=51)$.

Table 2 Fixed dosing administration schedules described in randomized controlled trials planning to assess the effects of hydroxychloroquine or chloroquine in the treatment of COVID-19 ( $\mathrm{n}=19)$.

Table 3 Variable dosing administration schedules described in randomized controlled trials planning to assess the effects of hydroxychloroquine or chloroquine in the treatment of COVID-19 ( $\mathrm{n}=20)$. 
Table 4 Efficacy and safety outcomes described in randomized controlled trials planning to assess the effects of hydroxychloroquine or chloroquine in the treatment of COVID-19 ( $\mathrm{n}=51)$.

Figure 1 Flow diagram illustrating the selection of the clinical trial registers available on the

the International Clinical Trials Registry Platform(ICTRP)onApril8, 2020at10:30GMT-6

Figure 2 Efficacy outcomes among randomized controlled trials of hydroxychloroquine or chloroquine for the treatment of COVID-19.

Figure 3 Safety outcomes among randomized controlled trials of hydroxychloroquine or chloroquine for the treatment of COVID-19.

Supplementary material

\section{Hosted file}

Table 1.docx available at https://authorea.com/users/324527/articles/452705-efficacy-andsafety-outcomes-in-randomized-controlled-trials-investigating-hydroxychloroquine-forcovid-19

\section{Hosted file}

Table 2.docx available at https://authorea.com/users/324527/articles/452705-efficacy-andsafety-outcomes-in-randomized-controlled-trials-investigating-hydroxychloroquine-forcovid-19

\section{Hosted file}

Table 3.docx available at https://authorea.com/users/324527/articles/452705-efficacy-andsafety-outcomes-in-randomized-controlled-trials-investigating-hydroxychloroquine-forcovid-19

\section{Hosted file}

Table 4.docx available at https://authorea.com/users/324527/articles/452705-efficacy-andsafety-outcomes-in-randomized-controlled-trials-investigating-hydroxychloroquine-forcovid-19 
Figure 1 Flow diagram illustrating the selection of the clinical trial registers available on the International Clinical Trials Registry Platform (ICTRP) on April $8^{\text {th }}, 2020$ at 10:30 GMT-6.

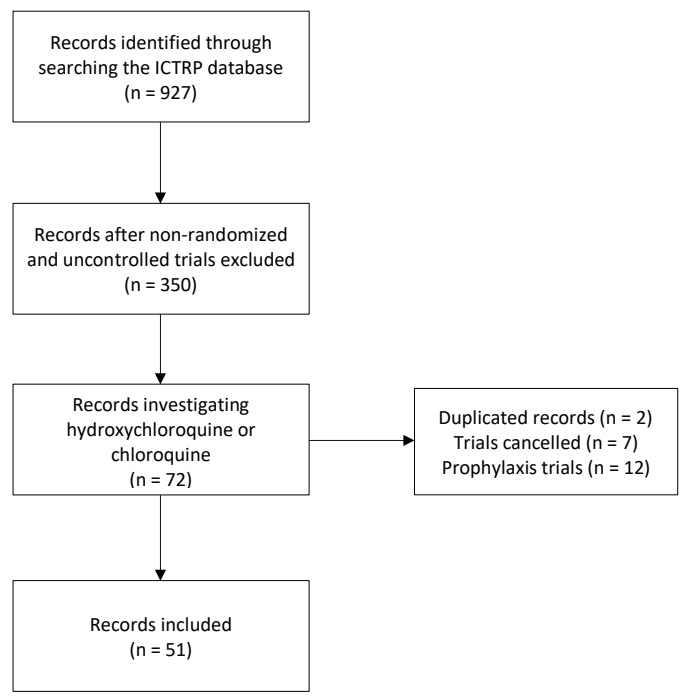


Figure 2 Efficacy outcomes among randomized controlled trials of hydroxychloroquine or chloroquine for the treatment of COVID-19.

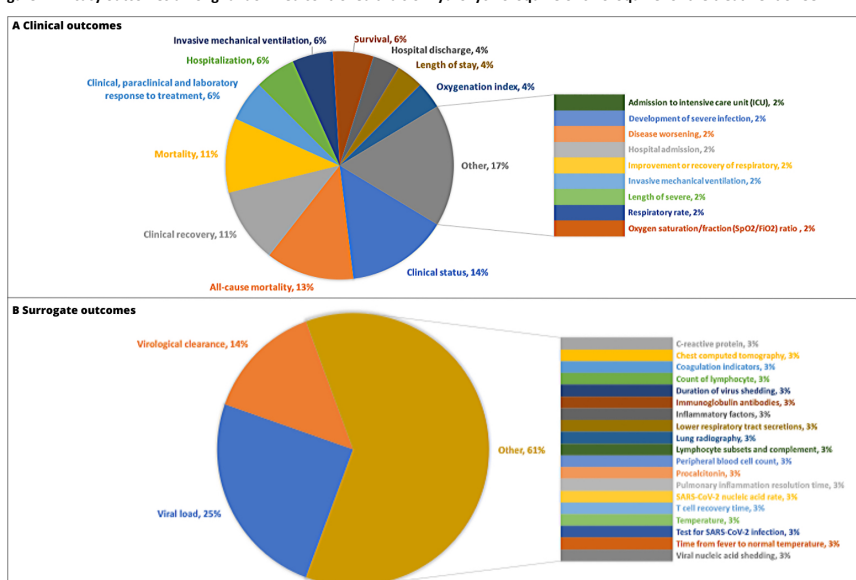

SARS-CoV-2: severe acute respiratory syndrome coronavirus 2. Unique outcomes described in the protocol of the randomized controlled trials; one trial could 15 protocols of clinical trials. 
Figure 3 Safety outcomes among randomized controlled trials of hydroxychloroquine or chloroquine for the treatment of COVID-19.

A Safety outcomes described in the protocol of at least two trials

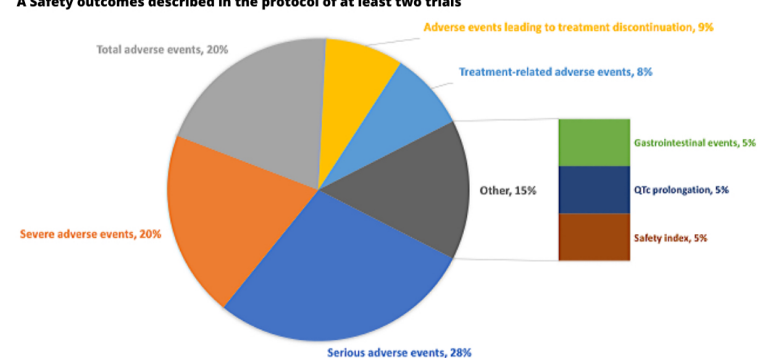

B Safety outcomes reported in the only one clinical trials protocol

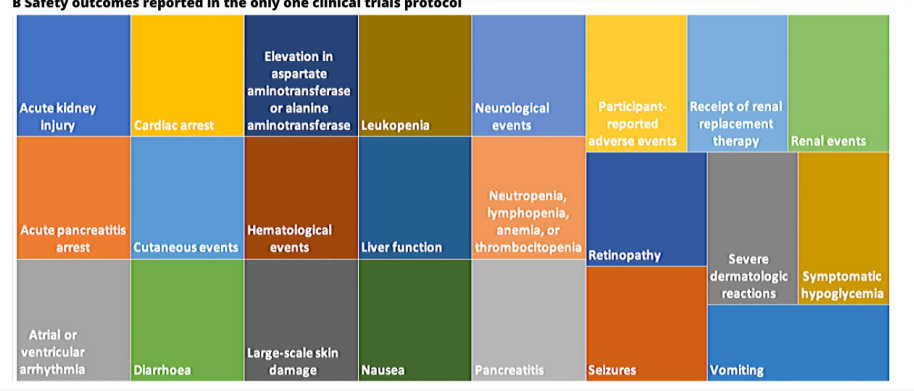

Unique outcomes described in the protocol of the randomized controlled trials; one trial could describe more than one outcome. A: 8 different safety outcomes among 27 protocols of clinical trials describing the assessment of at least on safety outcome; B 23 outcomes reported by only one the protocols of clinical trials describing the assessment of at least on safety outcome. 